\title{
Delivery of a DNA Vaccine for Alzheimer's Disease by Electroporation versus Gene Gun Generates Potent and Similar Immune Responses
}

\author{
Hayk Davtyan ${ }^{a, b} \quad$ Anahit Ghochikyan $^{a}$ Nina Movsesyan ${ }^{b}$ Barry Ellefsen ${ }^{d}$ \\ Irina Petrushinab David H. Cribbs ${ }^{b, c}$ Drew Hannamand Claire F. Evans ${ }^{d}$ \\ Michael G. Agadjanyan a, b \\ a Department of Molecular Immunology, Institute for Molecular Medicine, Huntington Beach, Calif., b Institute \\ for Memory Impairments and Neurological Disorders and ' Department of Neurology, University of California, \\ Irvine, Calif., and ${ }^{\mathrm{d} I c h o r ~ M e d i c a l ~ S y s t e m s, ~ S a n ~ D i e g o, ~ C a l i f ., ~ U S A ~}$
}

\section{Key Words}

Alzheimer's disease $\cdot$ DNA epitope vaccine $\cdot$

Electroporation $\cdot$ Gene gun $\cdot$ Immune responses

\begin{abstract}
Background: Induction of a humoral response against amyloid- $\beta$ peptide may be beneficial for Alzheimer's disease (AD) patients and may alleviate the onset and progression of AD. DNA-based vaccination provides a unique alternative method of immunization for treatment and prevention of AD. Currently, the two major delivery methods used for enhancing DNA uptake and immune responses to DNA vaccines in humans are electroporation (EP) and gene gun (GG). Objective: The goal of this translational study was to evaluate the efficacy of an AD DNA epitope vaccine (DepVac) delivered intramuscularly by EP or intradermally by GG. Methods: Humoral and cellular immune responses to immunization with DepVac were evaluated by ELISA and ELISPOT, respectively. Functional activity of the antibodies was also assessed. $\boldsymbol{R e}$ sults: EP- and GG-mediated immunizations with DepVac induced similar anti-amyloid- $\beta(A \beta)$ antibody and $T$ cell responses. Anti-A $\beta$ antibodies bound to amyloid plaques in $A D$ brain tissue and to toxic forms of $A \beta_{42}$ peptide. Conclusion: Both delivery methods are effective at promoting potent antibodies specific for $A \beta$.

Copyright $\odot 2012$ S. Karger AG, Basel
\end{abstract}

\section{Introduction}

Data from preclinical studies and clinical trials indicate that high titers of anti-amyloid- $\beta$ (A $\beta$ ) antibodies may prevent the accumulation of toxic forms of $A \beta$ peptide and can be beneficial to Alzheimer's disease (AD) patients [1-5]. However, in one clinical trial, immunization with an adjuvant $A \beta$ peptide vaccine resulted in meningoencephalitis in $6 \%$ of subjects, likely due to the generation of autoreactive T cells [5-7]. Previously, we suggested that an epitope vaccine composed of a self-B cell epitope of $A \beta$ peptide and a foreign $T$ helper cell epitope would be safe in humans since it should reduce the risk of an adverse T-cell-mediated autoimmune response while inducing a strong antibody response to $A \beta$. Using this strategy, we have developed different epitope vaccines based on peptide, recombinant protein or DNA [8-10]. Our DNA-based epitope vaccine (DepVac), composed of three copies of a short $A \beta B$ cell epitope fused with the foreign universal Th cell epitope, PADRE, and macrophage-derived chemokine, was immunogenic and safe in mice [8]. However, although DNA vaccines have

Hayk Davtyan and Anahit Ghochikyan contributed equally to this work.

\section{KARGER \\ Fax +41613061234 \\ E-Mail karger@karger.ch}

www.karger.com
(C) 2012 S. Karger AG, Basel

$1660-2854 / 12 / 0104-0261 \$ 38.00 / 0$

Accessible online at:

www.karger.com/ndd
Dr. Michael G. Agadjanyan

The Institute for Molecular Medicine

Huntington Beach, CA 92647 (USA)

Tel. +1 7145967821

E-Mail magadjanyan@immed.org 
been used to induce immune responses in small animal models for more than a decade, low immunogenicity in large animals and humans has limited their utility.

Accordingly, successful translation of a DNA vaccine to the clinical setting requires a suitable method for effective intracellular delivery. Electroporation (EP)- and gene gun (GG)-mediated administration are the most effective physical methods for delivering DNA plasmids in vivo and both are currently being tested in clinical trials [11, 12]. GG is a needle-free ballistic device which delivers gold beads coated with plasmid DNA into the epidermal layer of skin and drives strong immune response to DNA vaccines in animals [8] and humans [12]. EP is a technique for intracellular delivery based on the brief application of electrical fields in the target region of tissue. EP delivery of DNA vaccines enhances immune responses by several orders of magnitude compared to conventional injection in animal models [13] and humans [11]. A previous study demonstrated superior $T$ cell responses and tumor control with EP delivery compared to GG [14]. The goal of the current study was to evaluate the efficacy of antibody responses to DepVac delivered intramuscularly by EP or intradermally by GG. The results indicate that DepVac delivered by both methods induced potent titers of anti-A $\beta$ antibodies, as well as strong cellular responses against the foreign Th epitope, PADRE, but not self- $A \beta_{40}$.

\section{Animals and Methods}

Mice, DNA Immunizations, Detection of Humoral and Cellular Immune Responses and Functional Activity of Antibodies

C57BL/6 mice (5-6 weeks old; Jackson Laboratory, Calif., USA) were immunized on days 0,14 and 28 with $10 \mu \mathrm{g} /$ mouse DepVac [8] or noncoding control vector in a single anterior tibial muscle using the TDS-IM EP system (Ichor Medical Systems, Calif., USA) as described in Luxembourg et al. [15] or by intradermal delivery with three shots into the skin of the abdomen with GG bombardment (Bio-Rad, Calif., USA) $[8,10]$. Concentrations of anti-A $\beta$ antibodies and their isotype profiles were measured in sera collected 12 days after each administration as described previously $[8,10]$. To detect binding of immune sera to different forms of $A \beta_{42}$ peptide, a dot blot assay was run as described in Petrushina et al. [9]. To detect binding of immune sera to native amyloid (plaques), immunohistochemistry was performed on brain sections from a control or AD patient obtained from the University of California, Irvine, Calif., USA [8-10]. T cell proliferation was analyzed in splenocyte cultures restimulated in vitro with $10 \mu \mathrm{g} / \mathrm{ml}$ of PADRE peptide, $A \beta_{40}$ peptide, or with an irrelevant peptide using a $\left[{ }^{3} \mathrm{H}\right]$ thymidine incorporation assay; stimulation index was calculated as described earlier [10]. ELISPOT assay was used to determine the number of antigen-specific cells producing cytokines (IFN- $\gamma$ and IL-4) as described in Petrushina et al. [9].
Statistical Analysis

Prism 3.03 software (GraphPad Software, Calif., USA) was applied in the statistical analysis of the data. Statistically significant differences were examined using a t test and $\mathrm{p}<0.05$ was considered significant.

\section{Results and Discussion}

\section{DepVac Induces Strong and Therapeutically Potent}

Anti-A $\beta$ Antibodies in Mice Immunized with TDS-IM and $G G$

In order to select a suitable device for delivering DepVac to humans, we evaluated the efficacy of DepVac after EP-mediated intramuscular delivery and compared it to that with intradermal delivery by GG. Experimental and control animals received three immunizations and the kinetics of anti-A $\beta$ antibody responses were analyzed in individual sera collected from mice after each injection (fig. 1a). The results demonstrated that both methods are effective in inducing strong humoral immune responses. The level of anti-A $\beta$ antibodies after EP versus GG delivery was equivalent; after the third immunization, concentrations of antibodies were $145.1 \pm 69.2$ and $167.9 \pm$ $57.3 \mu \mathrm{g} / \mathrm{ml}$, respectively. Of note, intradermal and intramuscular immunizations of mice with DepVac without using any delivery device generated low titers of anti-A $\beta$ antibodies: $0.84 \pm 1.4$ and $21.3 \pm 17.5 \mu \mathrm{g} / \mathrm{ml}$, respectively. No anti-A $\beta$ response was seen in mice immunized with noncoding vector. Interestingly, EP-mediated vaccination induced predominantly IgG2a $\mathrm{a}^{\mathrm{b}}$ isotypes of antibodies (IgG1/IgG2ab ratio was 0.61 ), whereas the antibodies produced after GG vaccination were mostly of the IgG1 isotype (IgG1/IgG2 $\mathrm{a}^{\mathrm{b}}$ ratio was 11.87) (fig. 1b). These data were in accordance with other reports $[8,9$, $16,17]$, which indicates that these techniques may differ in activation of Th1 and Th2 pathways. One possible explanation could be connected with differential delivery of DNA. In case of muscle immunization, CpG motifs on plasmid backbone may interact with receptors in APC located in a target tissue and induce proinflammatory cytokines and Th1 type of immune response [18], while GG inoculation delivers DNA directly into the nucleus bypassing CpG receptors. Other explanations for these differences are also possible.

To demonstrate the functional activity of the anti-A $\beta$ antibodies, we analyzed binding of antisera to $A \beta$ plaques in brain tissues from an $\mathrm{AD}$ case. The immune sera from the EP and GG delivery groups bound to $\beta$-amyloid plaques and this binding was specific since it was blocked 


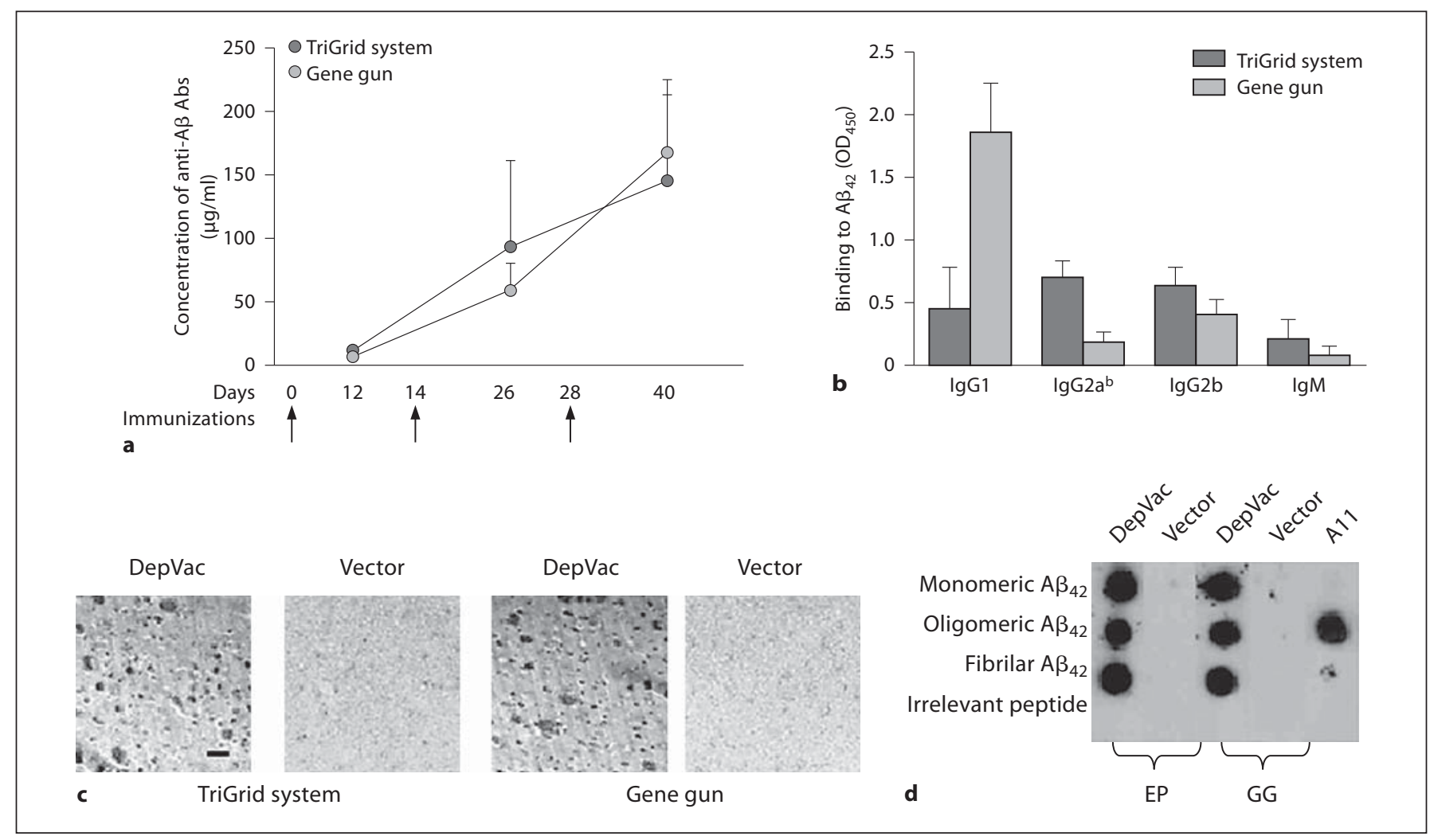

Fig. 1. DepVac delivered by EP or GG induces equivalent anti-A $\beta$ responses ( $n=8$ per group). Anti-A $\beta$ antibody concentrations were calculated by ELISA using a calibration curve generated with anti-A $\beta$ 6E10 monoclonal antibody (Signet, Dedham, Mass., USA) (a). DepVac delivered by EP induces antibody predominantly of IgG2ab isotype, whereas GG delivery induces predominant- ly IgG1 isotype (sera were used at dilution 1:500) (b). Immune, but not control sera bind to amyloid plaques in the brain section of the cortical region from an AD case (magnification $\times 10$, scale bar $100 \mu \mathrm{m})(\mathbf{c})$, and to all species of $A \beta_{42}$ peptide in dot blot (positive control A11 antibody binds to oligomers only) (d). by preabsorption of antisera with $A \beta_{42}$ peptide (data not shown) and was not observed with control sera (fig. 1c). Currently, it is thought that oligomers are the most toxic forms of $\mathrm{A} \beta$ responsible for disrupting neuronal functions and inducing cognitive decline in $\mathrm{AD}$ [1]. Therefore, we analyzed binding of immune and control sera to various forms of amyloid and demonstrated that only immune sera bound to monomeric, oligomeric and fibrillar $A \beta_{42}$ peptide (fig. 1d).

\section{EP- and GG-Mediated Immunizations with DepVac \\ Induce Strong Cellular Immune Responses}

To determine if enhancement of antibody responses after DepVac immunization by TDS-IM and GG is tied to higher $\mathrm{T}$ helper cell activation, we analyzed $\mathrm{T}$ cell responses in vaccinated mice. ELISPOT assay demonstrated that EP and GG delivery methods induced activation of similar numbers of IFN- $\gamma$ and IL- 4 producing cells af- ter restimulation of immune splenocytes with PADRE peptide, which were significantly higher compared with control groups ( $\mathrm{p} \leq 0.001$ and $\mathrm{p} \leq 0.01$, respectively) (fig. 2a). We also evaluated antigen-specific T cell activation by measuring $\mathrm{T}$ cell proliferation in splenocyte cultures restimulated with PADRE or irrelevant peptide. Data generated in this proliferation assay (fig. 2b) were in accordance with ELISPOT data presented in figure $2 \mathrm{a}$. Of note, as we expected from our previous studies $[8,9]$, an$\mathrm{ti}-\mathrm{A} \beta \mathrm{T}$ cell responses were not detected in immune splenocyte cultures (data not shown).

In conclusion, this study demonstrated that EP and GG delivery of DepVac were equally effective in generating anti-A $\beta$ humoral and anti-PADRE cellular immune responses, although the antibody isotype responses were significantly different. With GG delivery, a much smaller dose range can be evaluated than with intramuscular EP since only a limited amount of DNA can be loaded on the gold 
Fig. 2. DepVac delivered by TDS-IM or GG induces similar levels of cellular responses measured by ELISPOT assay $(\mathbf{a})$, and $\left[{ }^{3} \mathrm{H}\right]$ thymidine incorporation assay (b). Activation of splenocytes with the Th epitope PADRE was significantly higher in DepVac-immunized mice compared with vector-immunized groups (** $\mathrm{p} \leq 0.01$; ${ }^{* * *} \mathrm{p} \leq 0.001, \mathrm{n}=8$ per group).

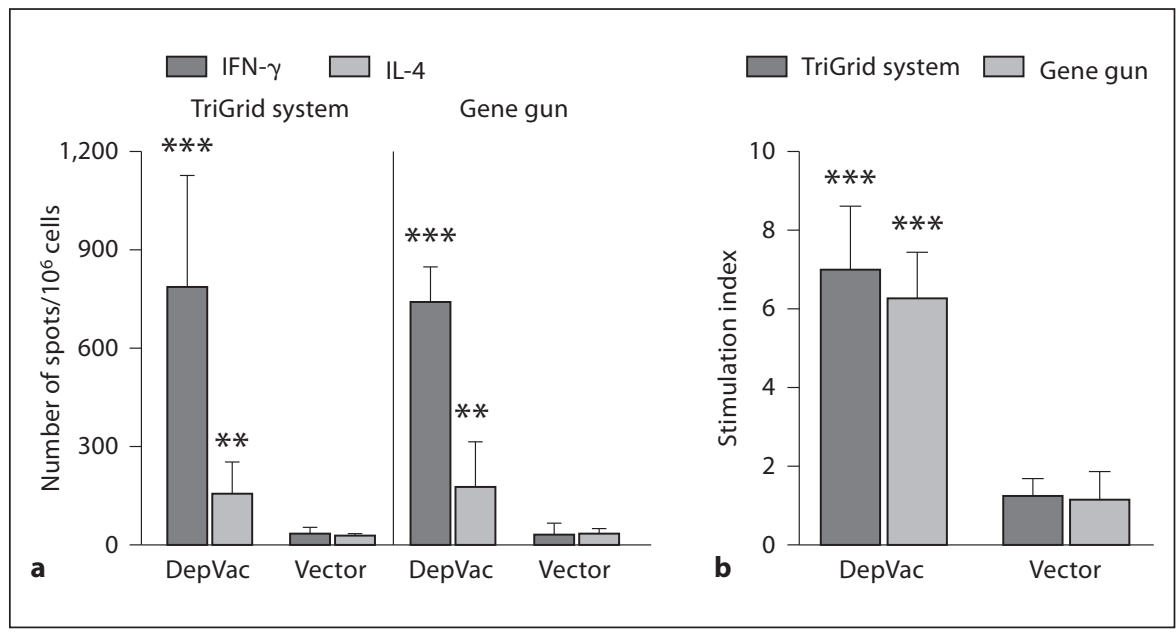

beads; thus, multisite administrations may be required in humans to achieve target responses. Therefore, based on the data presented here plus the larger potential dosing range provided by intramuscular EP delivery, we initiated preclinical testing of DepVac in rabbits and nonhuman primates using EP. If safe and effective in these species, the vaccine will be evaluated in a human clinical trial.

\section{Acknowledgments}

This work was supported by funding from NIH (AG-20241, NS-50895, NS-065518 and NS-057395) and Alzheimer's Association (IIRG-0728314). H.D. and N.M. were supported by NIA training grant AG000096. AD case tissues were provided by UCI ADRC (grant P50-AG16573).

\section{References}

1 Agadjanyan M, Cribbs D: Active and passive Abeta-immunotherapy: preclinical and clinical studies and future directions. Part 1. CNS Neurol Disord Drug Targets 2009;8:16.

2 Town T: Inflammation, immunity, and Alzheimer's disease. CNS Neurol Disord Drug Targets 2010;9:129-131.

3 Wilcock DM: The usefulness and challenges of transgenic mouse models in the study of Alzheimer's disease. CNS Neurol Disord Drug Targets 2010;9:386-394.

4 Lemere CA, Masliah E: Can Alzheimer disease be prevented by amyloid-beta immunotherapy? Nat Rev Neurol 2010;6:108-119.

5 Gilman S, Koller M, Black RS, Jenkins L, Griffith SG, et al: Clinical effects of Abeta immunization (AN1792) in patients with AD in an interrupted trial. Neurology 2005; 64:1553-1562.

6 6 Town T, Tan J, Flavell RA, Mullan M: T-cells in Alzheimer's disease. Neuromolecular Med 2005;7:255-264.

7 Boche D, Nicoll JA: The role of the immune system in clearance of Abeta from the brain. Brain Pathol 2008;18:267-278.
8 Movsesyan N, Ghochikyan A, Mkrtichyan $\mathrm{M}$, Petrushina I, Davtyan H, et al: Reducing AD-like pathology in 3xTg-AD mouse model by DNA epitope vaccine - A novel immunotherapeutic strategy. PLos ONE 2008; 3:e21-e24.

$\checkmark 9$ Petrushina I, Ghochikyan A, Mktrichyan M, Mamikonyan G, Movsesyan N, et al: Alzheimer's disease peptide epitope vaccine reduces insoluble but not soluble/oligomeric $\mathrm{A} \beta$ species in amyloid precursor protein transgenic mice. J Neurosci 2007;27:1272112731 .

10 Davtyan H, Mkrtichyan M, Movsesyan N, Petrushina I, Mamikonyan G, et al: DNA prime-protein boost increased the titer, avidity and persistence of anti-Abeta antibodies in wild-type mice. Gene Ther 2010;17: 261-271.

11 van Drunen Littel-van den Hurk S, Hannaman D: Electroporation for DNA immunization: clinical application. Expert Rev Vaccines 2010;9:503-517.

12 Jones S, Evans K, McElwaine-Johnn $\mathrm{H}$, Sharpe M, Oxford J, et al: DNA vaccination protects against an influenza challenge in a double-blind randomised placebo-controlled phase $1 \mathrm{~b}$ clinical trial. Vaccine 2009; 27:2506-2512.
$>13$ Livingston $\mathrm{BD}$, Little SF, Luxembourg A, Ellefsen B, Hannaman D: Comparative performance of a licensed anthrax vaccine versus electroporation based delivery of a PA encoding DNA vaccine in rhesus macaques. Vaccine 2010;28:1056-1061.

14 Best SR, Peng S, Juang CM, Hung CF, Hannaman D, et al: Administration of HPV DNA vaccine via electroporation elicits the strongest $\mathrm{CD} 8+\mathrm{T}$ cell immune responses compared to intramuscular injection and intradermal gene gun delivery. Vaccine 2009; 27:5450-5459.

15 Luxembourg A, Hannaman D, Nolan E, Ellefsen B, Nakamura G, et al: Potentiation of an anthrax DNA vaccine with electroporation. Vaccine 2008;26:5216-5222.

16 Town T, Tan J, Sansone N, Obregon D, Klein $\mathrm{T}$, et al: Characterization of murine immunoglobulin $\mathrm{G}$ antibodies against human amyloid- $\beta 1-42$. Neurosci Lett 2001;307:101-104.

-17 Town T, Vendrame M, Patel A, Poetter D, DelleDonne A, et al: Reduced Th1 and enhanced Th2 immunity after immunization with Alzheimer's beta-amyloid(1-42). J Neuroimmunol 2002;132:49-59.

18 Moreno S, Timon M: DNA vaccination: an immunological perspective. Immunologia 2004;123:41-55. 